\title{
Bacterial Isolates from Blind Tracheal Aspirates and Their Anti-Microbial Sensitivity: An Observational Study in ICU of a Tertiary Care Hospital in Bangladesh
}

Uzzwal Kumar Mallick*1, Md Sirajul Islam¹, Mohammad Asaduzzaman², Mohammad Abdullah Yusuf ${ }^{3}$, Mohammad Omar Faruq ${ }^{4}$

\begin{abstract}
:
Background: The number of organisms developing resistance to commonly used antibiotics is increasing day by day. The exact national scenario of antimicrobial sensitivity pattern is not well known in Bangladesh owing to the absence of proper guideline for prescribing antibiotics.

Aims: The aim of this study is to identify the group of organisms developing resistanceso that antibiotic policy can be formulated for the proper and effective use of antibiotics.

Settings and Design: This observational study was conducted for a period of 1 year from January 2018 to December 2018 in a tertiary care hospital in Bangladesh.

Materials and methods: This descriptive cross-sectional study was conducted in the Department of Microbiology, National Institute of Neurosciences and Hospital, Dhaka, Bangladesh from January 2018 to December 2018, using the convenient sampling technique. Tracheal secretions from patients in the intensive care unit (ICU), tested in Department of Microbiology, National Institute of Neurosciences and Hospital, were included in the study. The culture was done on blood and MacConkey agar and the sensitivity pattern was performed on Muller Hinton agar. Data were analyzed using SPSS version 23.0.

Results: Out of the microorganisms isolated from positive growth cultures, Acinetobacter (57.8\%) was the most common isolate followed by Klebsiella (22.9\%). Acinetobacter, pseudomonas and klebsiella had good sensitivity to colistin( $87.80 \%, 82.40 \%$ and $77.80 \%$ respectively), where as they showed less sensitivity to higher generationcephalosporin, penicillin and aminoglycosides.

Conclusion: The commonest organism which was isolated from the endotracheal aspirate cultures were Acinetobacter, followed by Pseudomonas and Klebsiella and antimicrobial susceptibility testing showed sensitivity to Colistin, Tazobactam/piperacillin, Meropenem and aztreonam. Whereas coagulase negative staphylococci was isolated only in minority cases with highest sensitivity to vancomycin and linezolid.
\end{abstract}

Keywords: Gram-negative bacteria (GNB), Drug sensitivity, Tracheal aspirate.

\section{Introduction:}

An intensive care unit (ICU) provides the critical care and life support for acutely ill and injured patients in a specialized

1 Assistant professor, Department of Critical Care Medicine, National Institute of Neurosciences \& Hospital, Dhaka, Bangladesh;

2 Assistant Professor,Critical Care, Kuwait Bangladesh Friendship Hospital, Uttara, Dhaka, Bangladesh.

3 Assistant Professor,Department of Microbiology, National Institute of Neurosciences \& Hospital, Dhaka, Bangladesh

4 Professor andChief Consultant, General ICU and Emergency Dept., United Hospital, Dhaka, Bangladesh.

* Corresponding Author:

Dr. Uzzwal Kumar Mallick, MD (CCM)

Assistant Professor

Dept. of Critical Care Medicine

National Institute of Neurosciences and Hospital

Dhaka, Bangladesh

Cell: +8801712715180

E-mail: ukm1980@gmail.com hospital. Hospital acquired infection (HAI) are the major cause of mortality and morbidity among the intubated patients in ICU. It is reported that mortality and morbidity rate by HAI is more among intubated patients in ICU (50\%) than among patients in general wards $(5 \%-10 \%) .{ }^{1}$

Equipment frequently used in ICU such as endotracheal tube, intra venous catheter etc. are mostly responsible for HAI. ${ }^{2}$ Tracheal intubation also causes colonization in trachea by different bacteria that may be responsible for increases the risk of mortality due to superinfections. ${ }^{3}$ This situation is causing concern regarding the rise of bacterial resistance to various antimicrobial agents and is becoming a major public health issue. Now a days, the major concern is inappropriate treatment against MDR (Multi-drug resistance) GNB (Gram-negative bacteria) due to unavailability of newer antibiotics. $^{4,5}$ As a result, there is reemergence of older antibiotics like colistin. ${ }^{6,7,8}$ The excessive and indiscriminate use of broad-spectrum antibiotics has led to the development of these resilient microbes which are difficult to treat ${ }^{9}$.

The aim of this study was to identify the common pathogens in tracheal secretions on patients with endotracheal tube in 
situ and to study the patterns of their sensitivity and resistance to various antibiotics, which can serve as guidelines to physicians for empirical treatment with proper antibiotics.To formulate an antibiotic policy for the ICU, knowledge ofthe antibiotic susceptibility of the organisms isolated in theICU is essential. This also avoids unnecessary use of broad-spectrum antibiotics and prevents emergence of drug resistant bacterial strains. The data on the changing antibioticsusceptibility trends is important for infection controlactivities in ICU settings. Therefore, the present study wasundertaken to determine the antimicrobial resistance patternof bacterial isolates from National Institute of Neurosciences and Hospital over a 1-year period. The results of this study is expected to help clinicians to plan the antibiotic guidelines aswell as antibiotic cycling in ICU settings.

\section{Material and Methods}

This descriptive cross-sectional study was conducted in the Department of Microbiology, National Institute of Neurosciences and Hospital, Dhaka, Bangladesh from January 2018 to December 2018. All patients with endotracheal tube in situ whose tracheal secretion samples obtained from blind tracheal aspiration were tested in the department of Microbiology, National Institute of Neurosciences and Hospital, were included in the study. In total, 332 samples were collected, regardless of age and gender, by using the convenient sampling technique. Tracheal secretions from patients admitted in the intensive care unit (ICU) for more than 48 hours were obtained by sterile suctioning through an endotracheal tube and suction catheter tip. Samples were inoculated on agar plates. The culture was done on blood and MacConkey agar and was incubated at $37^{\circ} \mathrm{C}$ for 24 to 48 hours. Microbes were identified under a microscope by observing morphological characteristics after gram staining and applying biochemical tests. Antibiotic sensitivity pattern was done on Muller Hinton agar using the Kirby Bauer disk diffusion method. Antibiotic discs containing amikacin,amoxicillin, aztreonam, ceftazidime, ciprofloxacin, Levofloxacin, Co-trimoxazole, ceftriaxone, cefepime, meropenem, piperacillin + tazobactam, vancomycin, penicillin, gentamicin, linezolid, chloramphenicol, erythromycin, methicillin, ampicillin, and Colistin sulfate were obtained and used as per the manufacturer's instructions.

Data were entered and analyzed using the Statistical Package for Social Sciences (SPSS) v.23.0 (IBM, Armonk, US). Descriptive statistics were applied to find frequencies and percentages. Charts and tables were constructed.

Results: A total of 332 samples were collected during this study period. Positive growth was observed in $76.5 \%$ samples (Figure-1). The gram-negative bacilli contributed a major number of isolates $(97.6 \%)$, the remaining nine $(1.2 \%)$ were caused by gram-positive cocci and candida was $1.2 \%$ (Figure-2). The gram-negative bacilli such as Acinetobacter (57.8\%) was the most common isolate followed by Klebsiella (22.9\%). Acinetobacter, pseudomonas and klebsiella had good sensitivity to colistin $(87.80 \%, 82.40 \%$ and $77.80 \%$ respectively), where as they showed less sensitivity to higher generation cephalosporins, penicillin and aminoglycosides. The susceptibility pattern of Gram-negative bacilliare shown in Figure-3 and susceptibility pattern of Gram-positive staphylococcus is shown in Figure-4 shows good sensitivity to vancomycin and linezolid ( $82.20 \%$ and $87.80 \%$ respectively) and less sensitivity to cephalosporine, quinolones and co-trimoxazole.

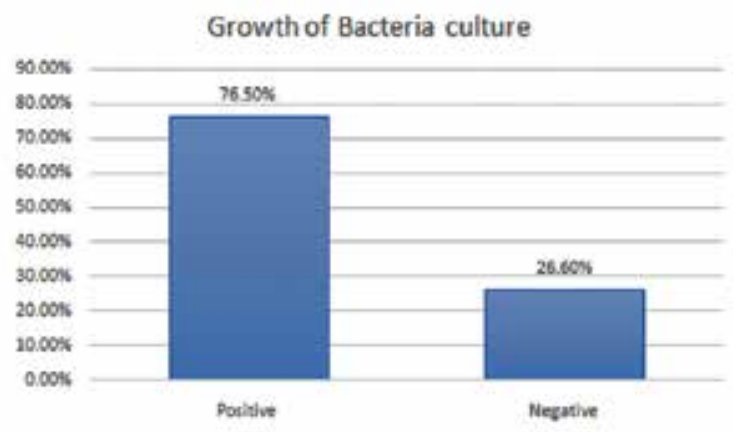

Fig 1

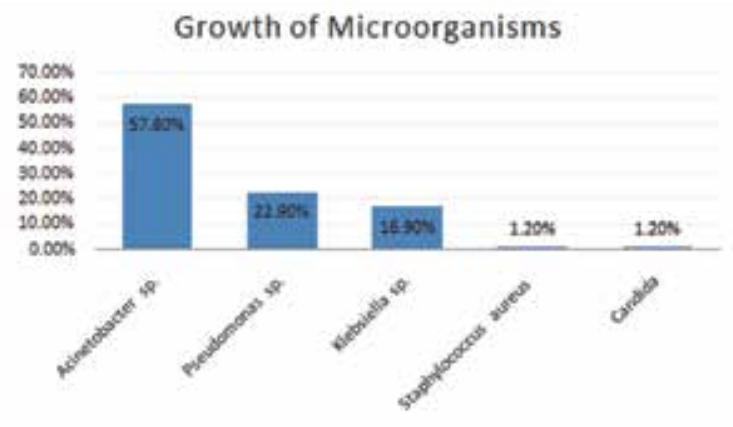

Fig 2

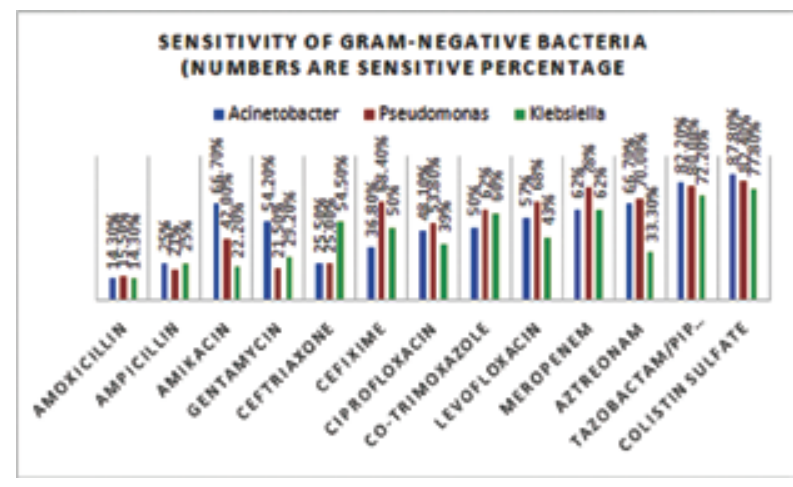

Fig 3

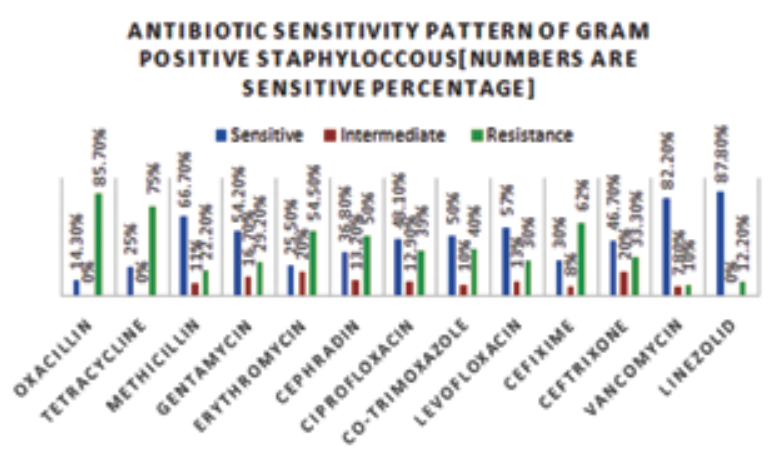

Fig 4 
Table 1

\begin{tabular}{|c|c|c|c|}
\hline References & Acinetobacter & Pseudomonas & Klebsiella \\
\hline \multicolumn{4}{|l|}{ Our study (2018) } \\
\hline Bacteria Positivity & $57.8 \%$ & $22.9 \%$ & $16.9 \%$ \\
\hline \multicolumn{4}{|l|}{ Antibiotic Sensitivity } \\
\hline Colistin & $87.8 \%$ & $84.3 \%$ & $77.8 \%$ \\
\hline Tazobactam/ piperacillin & $82.2 \%$ & $80 \%$ & $72.2 \%$ \\
\hline Carbapenem & $62 \%$ & $70.4 \%$ & $78 \%$ \\
\hline Amikacin & $66.7 \%$ & $42 \%$ & $22.2 \%$ \\
\hline
\end{tabular}

Karim et al.Bangladesh Critical Care Journal,2019. 7(2), 73-76. ${ }^{20}$

Bacteria Positivity

Antibiotic Sensitivity

Colistin

Tazobactam/ piperacillin

Carbapenem

Amikacin

Mallick et al Bangladesh Critical Care Journal, 2015. 3(1), 9-13 ${ }^{13}$

Bacteria Positivity

$38-66.6 \%$

Antibiotic Sensitivity

Colistin

Tazobactam/piperacillin

Carbapenem

Amikacin

Ahsan et al. Bangladesh Crit Care J September 2016; 4 (2): 69-73 ${ }^{12}$

Bacteria Positivity

Antibiotic Sensitivity

Colistin

Tazobactam/piperacillin

Carbapenem

Amikacin

Jamil et al. Journal of Medicine, 2016. 17(2), 91-94. ${ }^{21}$

Bacteria Positivity

$38-61 \%$

$38-42 \%$

$42 \%$

$30 \%$

$00 \%$

$62 \%$

$18 \%$

$45 \%$

$37.5 \%$

$100 \%$

$16.2 \%$

$13.1 \%$

$10.1 \%$

$64 \%$

Antibiotic Sensitivity

Colistin

Tazobactam/ piperacillin

Carbapenem

Amikacin
$98.6 \%$

$11.5 \%$

$17.4 \%$

$22 \%$
$21.05 \%$

$10.05 \%$

$60 \%$

$80 \%$

$80 \%$

$40 \%$
$20-42 \% \quad 15-16 \%$

$$
42 \%
$$

$15 \%$

$15 \%$

$15 \%$

$7-15 \%$

$15 \%$

$7-15 \%$

$7 \%$

$22.7 \%$

$13.6 \%$

$100 \%$

$100 \%$

$37.8 \%$

$12.8 \%$

$10.1 \%$

$40 \%$

$19.2 \%$

$30 \%$

$15 \%$

$9 \%$

$00 \%$

$43.3 \%$

$27.8 \%$

$29.1 \%$

$48 \%$

$19.2 \%$ 


\section{Discussion}

According to literature the rate of nosocomial infections are increasing in the patients admitted in the ICU due to excessive invasive procedures performed including artificial ventilator support $^{10}$. Bacterial sensitivity to conventional antibiotics are decreasing day by day ${ }^{11}$.In our study, percentage of samples showing positive growth in our study was $76.5 \%$. In a study conducted by Hassaan et al., the positive samples were $72.3 \%{ }^{12}$. In a study conducted in the setting of Pakistan by Malik et al., the positive cultures came out to be $83 \%{ }^{13}$. The lower positive growth in our study can be attributed to the better infection control measures in the ICU setup of our hospital. However, the convenient sampling technique used in our study might be a limiting factor for the decreased percentage of positive growth.

In our study, gram-negative bacilli were more common causative agents $(97.6 \%)$ as compared to gram-positive cocci, which were $1.2 \%$ of the total positive cultures. Another research in Bangladeshdone by Ahsan et al. and Mallick et al., in which the gram-negative bacilli were $76.13 \%$ and $86 \%$ respectively. ${ }^{12,13}$ (Table-1). This can be attributed to the fact that the majority of the nosocomial infections are caused by gram-negative bacteria which are more dangerous and difficult to treat. This calls for strict measures against the spread of gram-negative bacilli, especially in the ICU setting.Table 1shows comparison of our study with some similar studies done in Bangladesh

In our study, Acinetobacter (57.8\%) was the most common isolate. In a study by Ahsan et al., the commonest bacterium isolated from tracheal secretions was Acinetobacter $(37.5 \% \%)^{12}$ (Table- 1$)$ In a study by Mallick et al., Acinetobacter $(32.35 \%)$ was the most common isolate both in early and late onset Ventilator-associated pneumonia ${ }^{13}$ (Table-1). However, in one study by Shahunja et al. in Bangladesh, Klebsiella was the most common isolate (45\%), followed by Acinetobacter (36\%) and Pseudomonas $(14 \%)^{14}$. The rise in Acinetobacter in our study, especially in the ICU setup, can be attributed to the dramatic increase in the occurrence of multi-drug resistant isolates. In addition, this organism has the ability to survive in humid and dry conditions for longer periods, resulting in nosocomial outbreaks ${ }^{15}$.

In our study, Acinetobacter was most sensitive to Colistin (87.8\%) followed by Tazobactam/Piperacillin (82.2\%). A study by Rani et al. reported Acinetobacter $80 \%-90 \%$ sensitivity to Colistin ${ }^{16}$. In another study by Study done by Saha, et al showed Acinetobacter was highly sensitive only to colistin $98 \%$ but only $9-20 \%$ to Piperacillin and Klebsiella were moderate to highly sensitive to colistin $63.15 \%{ }^{17}$. In our study klebsiella sensitivity to colistin, Tazobactam/piperacillin, aztreonam and meropenem was $77.8 \%, 72.2 \%, 70 \%$ and $62 \%$ respectively. But in the study done by Haque L et al. klebsiella was more than $40 \%$ to $60 \%$ sensitivity to colistin, ciprofloxacin, amikacin and meropenem ${ }^{18}$. Our study revealed that pseudomonas had sensitivity to colistin, Tazobactam/piperacillin, aztreonam and meropenem and it was $82.4 \%, 80 \%, 78 \%$ and $70 \%$ respectively. But study done by Saha, et al showed pseudomonas sensitive to colistin, carbapenem were $100 \%$ and $50 \%-60 \%$ respectively ${ }^{17}$. Study done by Karim et al. and Jamil et all also showed near similar result ${ }^{20,21}$.

In our study high sensitivity of S. aureus was observed for linezolid (87.8\%) and vancomycin $(82.2 \%)$ showed less sensitivity to commonly used antibiotics such as gentamycin, erythromycin, levofloxacin, tetracycline and cephradine which is similar to study done by Gitau et $\mathrm{al}^{19}$. In our study candida was isolated from $1.2 \%$ of tracheal aspirate culture, which maybe due to the presence of underlying conditions like poor nutritional status, diabetes mellitus, use of steroid and broad-spectrum antibiotics. This may, however, also indicate an overuse of antibiotics.

Conclusion: The commonest organism which was isolated from the endotracheal aspirate cultures were Acinetobacter, followed by Pseudomonas and Klebsiella which were sensitive to colistin, meropenem, aztreonam and Tazobactam/piperacillin. Bacterial Sensitivity to major antibiotics are decreasing day by day and complicating empirical selection of antibiotics in the ICU. Our dataindicate an alarming pattern of poor antibiotic-sensitivity ofmajority of ICU isolates to most of broad-spectrum antibiotics. So, strategies must be taken to prevent the emergence of multidrug resistant bacteria in ICU immediately. These include: knowledge of the infection rates and common pathogens and judicious use of older and newer antimicrobial agent according to antibiogram. Our data will help clinicians in choosing appropriate empiric antibiotics to maximize the patient's chances of receiving early and effective therapy.

Study Limitation: Study samples obtained from blind tracheal aspirates often could not distinguish colonization from true infections. Consecutive three days sampling were not done in this study patients. Clinical corelation were not done. As such absence of clinical corelation could not point to colonization.

\section{References:}

1. Bagheri NS, Allegranzi B, Syed SB, Elli B, Pitter D. Health-care associated infection in Africa: a systematic review. Bull World Health Organ 2011; 89: 757-765.

2. Hoque L, Mostofa KSM, Ahmed Z. Isolation, identification and antimicrobial sensitivity patterns of bacterial isolates from tracheal aspirate of ICU patients of central Dhaka, Bangladesh. Int J Res App Natural and Social Sci., 2013; 1: 11-16.

3. Saha AK, Nandi S, Dhar P. Prevalence of bacterial isolates in endotracheal tube according to culture and sensitivity in patients of ICU of a tertiary medical college and hospital, Kolkata, West Bengal. Int J Contem Med Res., 2016; 3(6): 1775-1781.

4. Talbot GH, Bradley J, Edwards J, Gilbert D, Scheld M, Bartlett JG. Bad bugs need drugs: an update on the development pipeline from the antimicrobial availability task force of the Infectious Diseases Society of America. Clin Infect Dis., 2006; 42: 657-668. 
Bangladesh Crit Care J September 2020; 8 (2): 81-85

5. Li J, Nation RL, Turnidge JD, Milne RW, Coulthard K, Rayner CR et al. Colistin: the re-emerging antibiotic for multidrug-resistant gram-negative bacterial infections. Lancet Infect Dis., 2006; 6: 589-601.

6. Lim LM, Ly N, Anderson D, Yang JC, MacanderL,Jarkowski A. et al,. Resurgence of Colistin: A Review of Resistance, Toxicity, Pharmacodynamics, and Dosing Pharmacotherapy, 2010; 30(12): 1279-1291.

7. Falagas ME, Kasiakou SK, Tsiodras S, MichalopoulosA.The use of intravenous and aerosolized polymyxins for the treatment of infections in critically ill patients: a review of the recent literature. Clin Med Res., 2006; 4: 138-146.

8. Li J, Nation RL, Milne RW, Turnidge JD, CoulthardK.Evaluation of colistin as an agent against multi-resistant gram-negative bacteria. Int J Antimicrob Agents, 2005; 25: 11-25.

9. Dautzenberg MJ, Wekesa AN, Gniadkowski M, et al. The association between colonization with carbapenemase-producing enterobacteriaceae andoverallICUmortality:anobservationalcohort study.. Crit Care Med. 2015 Jun; 43:1170.

10. Pattanayak C, Patanaik SK, Datta PP, Panda P. A study on antibiotic sensitivity pattern of bacterial isolates in the intensive care unit of a tertiary care hospital in Eastern India. Int J Basic Clin Pharmacol. $2013 ; 2: 153-159$.

11. Kumarasamy KK, Toleman MA, Walsh TR, et al. Emergence of a new antibiotic resistance mechanism in India, Pakistan, and the UK: a molecular, biological, and epidemiological studyLancet Infect Dis. 2010; 10:597-602.

12. Ahsan ASMA, Barai L, Faruq MO, Fatema K, Ahmed F, Saha D, Saha M, Nazneen S, Hamid T, Zabeen N. Antibiotic Resistance Pattern among Bacteria causing Ventilator Associated Pneumonia in An Intensive Care Unit of Bangladesh. Bangladesh Critical Care Journal,2016; 4(2), 69-73.

13. Mallick UK, Faruq MO, Ahsan ASMA., Fatema K, Ahmed F., Asaduzzaman M., Islam M., \& Sultana A. (2015). Spectrum of Early Onset and Late Onset Ventilator Associated Pneumonia (VAP) in a Tertiary Care Hospital of Bangladesh: A Prospective Cohort Study. Bangladesh Critical Care Journal,2015 ; 3(1), 9-13

14. Shahunja K., Salam MA, Ahmed T., Bardhan P, Sarker S, Ashraf H, Faruque AS, Hossain MI, Islam MM, Das S, Sharifuzzaman M, Bin Shahid A S, Sarker MH, Chisti MJ. Bacterial Isolates from Tracheal Aspirates and their Anti-microbial Susceptibility in Mechanically-Ventilated Children with Pneumonia Admitted to an Urban Critical Care Ward. Bangladesh Critical Care Journal,2015. 2(2), 60-64.
15. Baraibar J, Correa H, Mariscal D, Gallego M, Vallés J, Rello J.Risk factors for infection by Acinetobacter baumannii in intubated patients with nosocomial pneumoniaChest. 1997;112:1050-1054.

16. Rani P, Latha MB, Reddy SG, Bilolikar AK. A study of Acinetobacter from various clinical specimens and its antibiotic sensitivity pattern in a tertiary care hospital. J Med Sci Res. 2015;3(4):162-165. doi: 10.17727/JMSR.

17. Saha AK, Nandi S, Dhar P. Prevalence of bacterial isolates in endotracheal tube according to culture and sensitivity in patients of intensive care unit of a tertiary medical college and hospital, Kolkata, West Bengal. International Journal of Contemporary Medical Research 2016;3(6):1775-1781.

18. Hoque L, Mostofa Kamal SM, Ahmed Z. Isolation, Identification and antimicrobial sensitivity patterns of bacterial isolates from tracheal aspirate of ICU patients of Central Dhaka, Bangladesh. Int $\mathrm{J}$ of Research in Applied, natural and Social Sciences. 2013; 1:11-16.

19. Gitau W,Masika M, Musyoki M, Museve B, Mutwiri T. Antimicrobial susceptibility pattern of Staphylococcus aureus isolates from clinical specimens at Kenyatta National Hospital Wilfred BMC Res Notes (2018)11:226

20. Karim MR, Mayedah R, Cader FA. Ventilator-associated pneumonia in Coronary Care Unit of a tertiary level hospital in Bangladesh: causative organisms and pattern of antibiotic sensitivity. Bangladesh Critical Care Journal. 2019; 7(2), 73-76.

21. Jamil SM, Faruq MO, Saleheen S, Biswas P, Hossain MS, Hossain S, Arifuzzaman M, Basu BK, Kabir A, Alam F , lsan SK. (2016). Microorganisms Profile and their Antimicrobial Resistance Pattern Isolated from the Lower Respiratory Tract of Mechanically Ventilated Patients in the Intensive Care Unit of A Tertiary Care Hospital in Dhaka. Journal of Medicine.2016; 17(2), 91-94. 